\title{
Weibull Strength Analysis of Pineapple Leaf Fiber
}

\author{
C.V. Sia ${ }^{1, a^{*}}$, J.S.Y. Wong ${ }^{1, b}$, S.K. Thangavelu, ${ }^{1, c}$, K.H. Chong ${ }^{1, d}$ \\ and A. Joseph $2, \mathrm{e}$ \\ ${ }^{1}$ Mechanical Engineering Department, Swinburne University of Technology (Sarawak Campus), \\ Kuching, Sarawak, Malaysia, 93350 \\ ${ }^{2}$ Electrical and Electronics Engineering Department, University Malaysia Sarawak, \\ Kota Samarahan, Sarawak, Malaysia, 94300 \\ acvsia@swinburne.edu.my, b100064569@students.swinburne.edu.my, \\ 'sthangavelu@swinburne.edu.my, dkchong@swinburne.edu.my, ejannie@unimas.my
}

Keywords: weibull, PALF, fiber strength, scale effect.

\begin{abstract}
Pineapple leave fiber (PALF) can be considered as one of the green materials to the industries, which is the potential to replace the non-renewable synthetic fiber. However, the high disparity in the mechanical properties of PALF becomes an issue in structural composite design. Hence, improved Weibull distribution is utilised to quantify the tensile strength variation of PALF in various gauge lengths. The single fiber tensile test was performed after the fiber surface treatment and fiber diameter scanning. The predicted PALF strength by applying the improved Weibull distribution incorporating with conical frustum model is well compromised with experimental data compared to the traditional Weibull model.
\end{abstract}

\section{Introduction}

All Over the past decades, conventional materials were replaced with bio-based composites in various engineering applications due to the benefits of cost reduction, ease of processing and productivity [1]. Furthermore, low cost, easily obtainable and high specific properties of natural fibers are currently receiving greater attention as the potential alternative reinforcement materials in composite [2].

Pineapple leaf fiber (PALF) is a smooth and glossy fiber, which easily to be extracted from pineapple leaf by water retting and scrapping process [3]. The cellulose content of PALF is higher than other natural fibers [4]. Dey et al. [5] reported that PALF has better mechanical characteristics with respect to jute fiber. However, the mechanical characteristics of PALFs are highly inconsistent [6-11]. Therefore, statistical methods need to be considered to evaluate the tensile strength of PALFs. Recently, Weibull distribution is extensively utilised to analyse the inconsistency of the fiber mechanical characteristics. The Weibull distribution method also used to evaluate the tensile strength variation of natural fibers, such as banana [12], oil palm [13], sisal [14], jute [15], palm leaf sheath [16] and flax [17].

\section{Weibull Distribution Analysis}

The strength distribution by traditional Weibull model is derived as following [18]:

$F_{f}=1-\exp \left[-\left(\frac{V}{V_{c}}\right)\left(\frac{\sigma}{\sigma_{c}}\right)^{\alpha}\right]$

where $F_{f}$ is fiber strength probability, $\alpha$ is Weibull modulus, $V$ is the fiber volume, $V_{c}$ is standard volume, $\sigma$ is fiber strength, and $\sigma c$ is characteristic strength.

However, Eq. 1 is not preferable for natural fiber strength prediction. The predicted strength of natural fiber was reported to vary significantly compared with the experimental data [19,20]. Hence, Gutans and Tamuzh [21] and Watson and Smith [22] proposed an improved Weibull distribution, in which adding power of $\gamma$ on $V / V_{c}$ in Eq. $1,0<\gamma<1$, where $\gamma$ is the shape parameter: 